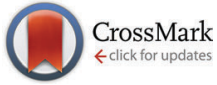

Cite this: Chem. Commun., 2015, 51,5264

Received 22nd January 2015, Accepted 2nd March 2015

DOI: $10.1039 / \mathrm{c5cc00644a}$

www.rsc.org/chemcomm

\title{
One-pot synthesis of doxorubicin-loaded multiresponsive nanogels based on hyperbranched polyglycerol $\dagger$
}

\author{
Ana Sousa-Herves, Stefanie Wedepohl and Marcelo Calderón*
}

\begin{abstract}
Doxorubicin-loaded nanogels with multiresponsive properties are prepared using hyperbranched polyglycerol as a biocompatible scaffold. The nanogels are synthesized in a single step combining free-radical polymerization and a mild nanoprecipitation technique. The nanogels respond to different biological stimuli such as low $\mathrm{pH}$ and reductive environments, resulting in a more efficient cell proliferation inhibition in A549 cells.
\end{abstract}

Recent advances in nanomedicine have prompted the need to develop delivery systems that can encapsulate a variety of novel therapeutics. The ideal drug delivery system should be biodegradable and biocompatible, target only the desired cells and tissue, and release their cargo at the intracellular space. ${ }^{1,2}$ Moreover, these nanocarriers should be smart so that they can deliver their payload at a well-defined time, place, or after a specific stimulus. ${ }^{3,4}$ Selected examples of biological stimuli include $\mathrm{pH}$, temperature, and redox microenvironments. ${ }^{3,5} \mathrm{pH}$-sensitiveness can be exploited for selective drug release at the slightly acidic $\mathrm{pH}$ of tumors and/or at the more acidic cellular compartments endosome/lysosome. Complementary, redox sensitivity can trigger the cytosolic release of drugs due to the different concentrations of glutathione (GSH) in extracellular and intracellular compartments.

Among the different nanostructures employed in drug delivery, polymeric nanogels (NGs) have recently attracted a great deal of attention. ${ }^{6-10}$ NGs are a type of crosslinked, nanometric-sized hydrogel particles. They have a tunable size and a large surface area for multivalent bioconjugation and can incorporate therapeutics in their interior network. The entrapment of bioactive molecules (including anticancer drugs, proteins, and nucleic acids) in NGs as well as their in vitro release has been extensively investigated. ${ }^{6-10}$ The advantages of NGs over other drug delivery systems include their large water content, soft nature, high loading capacity, and stability of the resulting dispersion. Moreover, NGs can be

Institut für Chemie und Biochemie, Freie Universität Berlin, Takustrasse 3, 14195 Berlin, Germany. E-mail: marcelo.calderon@fu-berlin.de

$\dagger$ Electronic supplementary information (ESI) available: Experimental section and supplemental figures. See DOI: 10.1039/c5cc00644a engineered to provide optimal loading and release of drugs. In this context, the groups of Haag and Calderón have recently reported the preparation of NGs using hyperbranched polyglycerol (hPG) as a biocompatible, multifunctional scaffold. ${ }^{11-13}$ It is noteworthy that $\mathrm{hPG}^{\mathbf{1 4 , 1 5}}$ has been shown to be even less cytotoxic than the structurally similar and FDA-approved polyethylene glycol. ${ }^{16,17}$ Although there are several reports on smart NGs, ${ }^{4,18,19}$ the preparation of multi-stimuli responsive NGs loaded with anticancer drugs ${ }^{11,20,21}$ is usually relatively complex and requires high shear stress (such as ultrasonication or high-pressure homogenization used in miniemulsion), multiple purification steps, and encapsulation of the drug after NG formation. ${ }^{22}$

Herein, we present an efficient one-pot approach for preparing multiresponsive NGs that have been loaded with the anticancer drug doxorubicin (DOX) and employ hPG as a biocompatible scaffold. We have incorporated a $\mathrm{pH}$-sensitive-monomer and a redox-responsive crosslinker into the NGs structure to give them stimuli-responsive properties. The NGs have been synthesized by free radical polymerization in combination with a nanoprecipitation ${ }^{23}$ technique to template NG formation during the polymerization process (Scheme 1). To the best of our knowledge, this is the first example of NG preparation by nanoprecipitation employing free radical polymerization.

First the bulk hydrogels were prepared in order to check the chemical compatibility and suitability of the system. The success of macrogel formation encouraged us to synthesize the gels with sizes in the nanometer scale. Unloaded NGs were prepared first in order to establish the synthetic conditions and to optimize the desired multiresponsive properties. Monomethyl oligo(ethylene glycol)acrylate (OEGA) and pH-responsive 2-(5,5-dimethyl-1,3dioxan-2-yloxy)ethyl acrylate DMDEA ${ }^{20,24}$ were selected as monomers. Redox-responsive bis(2-methacryloyl)oxyethyl disulfide (BMADS) was employed as the crosslinker while acrylated hPG (hPG-Ac, 35\% functionalization) was chosen as a macro-crosslinker (Scheme 1). NGs were synthesized by free radical polymerization using a nanoprecipitation approach to template gel formation. The nanoprecipitation method involves particle formation by injection of the monomers and crosslinker solution into a non-solvent, 

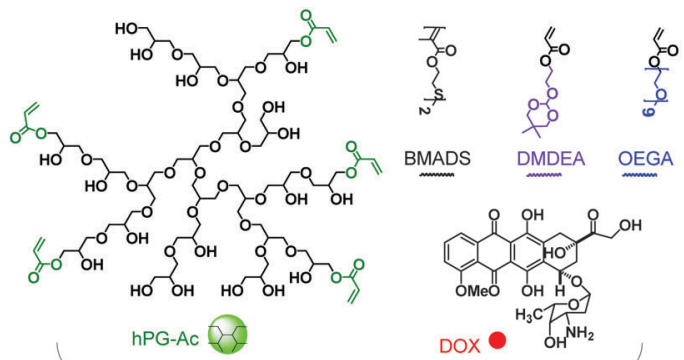

BMADS
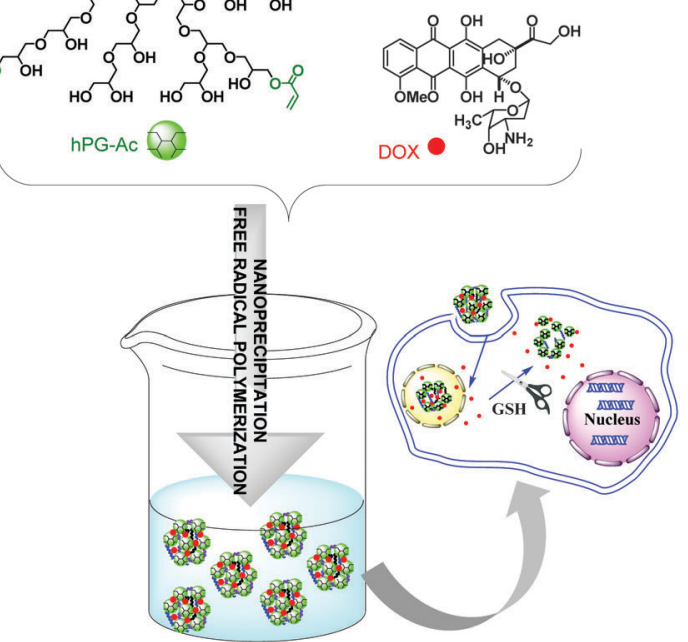

Scheme 1 Preparation of multiresponsive NGs and proposed mechanism of action.

and a simultaneous formation of a colloidal suspension caused by diffusion of the solvent into the non-solvent. ${ }^{23}$ This technique has been traditionally employed for the preparation of nanoparticles from previously synthesized polymers, in particular from poly(lactic acid) and its copolymers. More recently, we and others have exploited inverse nanoprecipitation in water/acetone $^{11,12}$ and thermo-nanoprecipitation ${ }^{13}$ for the preparation of NGs via chemical crosslinking of preformed polymeric scaffolds. In this work, nanoprecipitation was employed for the first time to prepare NGs comprising chain-growth polymerization.

Therefore hPG was highly functionalized with acrylate groups in order to obtain a derivative with similar solubility to hydrophobic DMDEA and BMADS. DMF was employed as the solvent and an aqueous solution of phosphate buffer ( $\mathrm{PB}, 50 \mathrm{mM} \mathrm{pH}$ 8) as the nonsolvent. Sodium dodecyl sulfate (SDS) was used as surfactant and AIBN as the organo-soluble initiator for the radical polymerization. The monomers, crosslinkers, and initiator were dissolved in DMF and subsequently precipitated into the non-solvent. The oxygen was then removed and the reaction temperature was increased to $65{ }^{\circ} \mathrm{C}$ to start the polymerization. The resulting NGs were extensively purified by dialysis in PB $10 \mathrm{mM} \mathrm{pH} 8$ and stored at $4{ }^{\circ} \mathrm{C}$. Detailed synthetic conditions are described in the ESI. $\dagger$

Several synthetic parameters were screened in order to obtain a suitable system for biological applications, i.e., with an adequate size, stability and low polydispersity (PDI). As a result, DMF and $\mathrm{MeOH}$ were tested as the solvent, whereby DMF turned out to be more suitable (in terms of particle size and stability) in a solvent/ non-solvent ratio of $1: 20$. The influence of monomers and crosslinkers concentration as well as the monomer's feed-ratio in the NGs sizes was also studied (Table S1, ESI $\dagger$ ). Interestingly, raising the hPG concentration from $12.5 \%$ to $25 \%$ (weight $\%$ ) only resulted in a small increase in the NG's size from 87 to $97 \mathrm{~nm}$, as measured
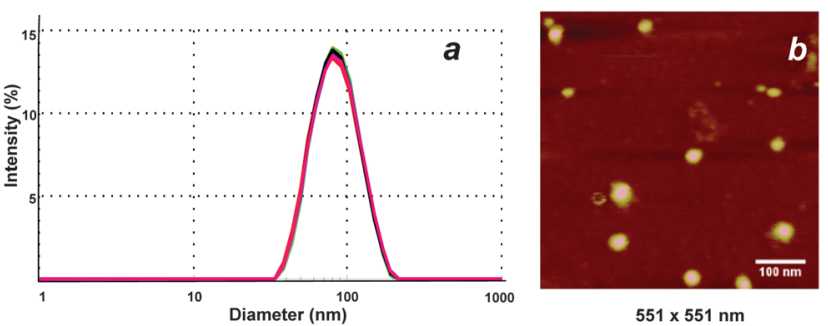

Fig. 1 (a) DLS intensity size distribution and (b) AFM of NG 3.

by dynamic light scattering (DLS). More importantly, employing the synthetic conditions reported in this work, NGs could not be obtained when hPG was not present. This suggests that the role of the hyperbranched macromolecule in the nanoprecipitation process is very important. In addition, a significant increase in the NG's size (87 vs. $176 \mathrm{~nm}$, by DLS) could be observed when the ratio between hydrophobic DMDEA and hydrophilic OEGA was increased. NG 3 in Table S1 (ESI $\dagger$ ) was selected as the optimal NG formulation because of its higher concentration and appropriate size. As shown in Fig. 1a, DLS measurements for NG 3 revealed a hydrodynamic size of $c a .87 \mathrm{~nm}$ and a low PDI (0.11). Atomic force microscopy (AFM) demonstrated the presence of spherical, uniformly distributed particles with a mean diameter of $45 \mathrm{~nm}$ (Fig. 1b). The smaller value observed in AFM can be attributed to the partial deswelling of the particles on the mica surface, as previously reported for other hydrophilic NGs. ${ }^{13,25}$

The progress of free radical polymerization could be easily monitored by the disappearance of the acrylate and methacrylate peaks (5.5-6.5 ppm) and the appearance of the peaks corresponding to the polymeric backbone in the ${ }^{1} \mathrm{H}$ NMR spectrum (Fig. S1, ESI $\dagger$ ). The OEGA peaks could be observed by NMR, which confirmed that OEGA did not diffuse to the aqueous phase during the polymerization. The hPG peaks could not be clearly seen because they overlapped with those of DMDEA and OEGA, but their presence within the NG is ensured by the fact that NGs do not form if hPG is not included in the formulation. As a comparison, ${ }^{1} \mathrm{H}$ NMR spectrum of NG 5 (lacking DMDEA monomer) is included in the ESI $\dagger$ (Fig. S2). In addition, FT-IR spectroscopy of the freeze-dried NGs supported the lack of acrylate groups in the NGs (Fig. S3, ESI $\dagger$ ).

The multiresponsive behavior of the NGs was studied by DLS and AFM. Lowering the $\mathrm{pH}$ from 8 to 5 resulted in a huge increase in the size of NG 3 (from 87 to $200 \mathrm{~nm}$ ), due to hydrolysis of the orthoester groups in DMDEA units, which led to more hydrophilic moieties and prompted the swelling of the NGs (Fig. 2a). The PDI of the hydrolyzed NGs was, nevertheless, quite low (0.16), probably because the hydrolysis of one of the monomers did not affect the integrity of the crosslinked polymeric network. AFM images of the hydrolyzed NGs further confirmed the increase in the mean size (166 nm, Fig. 2a). Notably, such an effect was not observed for the control NG 5, which lacks DMDEA monomer. In addition, the disappearance of the peak at $5.5 \mathrm{ppm}$ in the ${ }^{1} \mathrm{H} \mathrm{NMR}$, which corresponds to the orthoester group, proved the hydrolysis of the NGs.

Similarly, the presence of the reductive agent dithiothreitol (DTT) produced an important increase in the sizes and PDI of 

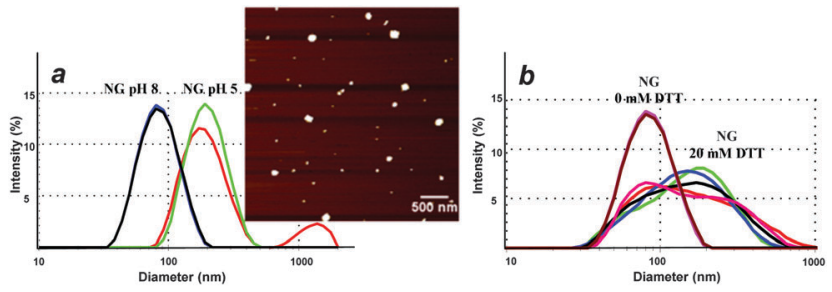

Fig. 2 (a) Intensity size distribution of $\mathrm{NG} 3$ at $\mathrm{pH} 8$ and at $\mathrm{pH}$ 5. AFM image of a hydrolyzed NG $(3.8 \times 3.8 \mu \mathrm{m})$. (b) Intensity size distribution of NG 3 (w/o DTT) and in the presence of $20 \mathrm{mM}$ DTT.

the NGs (183 nm, PDI 0.26, Fig. 2b), which can be attributed to reduction-cleavage of one of the crosslinkers' disulfide bonds. In this case, the higher PDI values arose from the destabilization of the polymeric network. It is important to note that NGs did not completely decompose because the presence of the macro-crosslinker hPG preserves part of the polymeric network. This feature (high increase in size and PDI) can be exploited to achieve post-endocytic nanogel destabilization and drug release, due to the large intra/extracellular redox gradient present in most cells. Again, control NG 7, which lacked BMADS, did not show any sign of destabilization when treated under identical circumstances. Remarkably, the combination of OEGA with more hydrophobic monomers is known to present thermoresponsive properties, and has been employed by several groups for the preparation of thermosensitive nanogels. ${ }^{20,26}$ Indeed, we observed an aggregation behavior for the NGs by DLS (presence of particles $>1000 \mathrm{~nm}$ ) when the temperature was increased from 25 to $55^{\circ} \mathrm{C}$. This process was completely reversible and the aggregates disappeared when the temperature was lowered back to $25{ }^{\circ} \mathrm{C}$. Unfortunately, the cloud point for the NGs could not be obtained by typical turbidity experiments at different temperatures. In order to deeply investigate the sensitivity of NG 3 towards temperature, we employed nanoparticle tracking analysis (NTA). NTA was performed in highly diluted solutions $\left(0.01-0.001 \mathrm{mg} \mathrm{mL}^{-1}\right)$, which at the same time confirmed the stability of the NGs upon dilution, an important fact when intravenous applications are considered. NTA measurements showed that the number of particles decreased by $40 \%$ when temperature was increased from 25 to $55{ }^{\circ} \mathrm{C}$ (from $3.62 \times$ $10^{8}$ particles per $\mathrm{mL}$ to $2.23 \times 10^{8}$ particles per $\mathrm{mL}$ ), however, the main size of the particles remained in the same size range (101 and $109 \mathrm{~nm}$, respectively). Taking into account the information obtained by DLS and NTA, we hypothesize that the NGs rapidly shrink when the temperature reaches a certain value, which leads to a reversible aggregation of the resulting smaller particles.

With a reliable and simple method for the preparation of multiresponsive NGs in hand, we decided to go a step further and incorporate the anticancer drug DOX during NG synthesis. NGs were basically prepared as described above but included non-protonated DOX in the DMF solution (detailed synthetic conditions in the ESI $\dagger$ ). Commonly employed DOX.HCl could not be incorporated due to hydrolysis of the NGs under acid conditions. Ideally, the drug would interact with macromolecular hPG and the monomers when injected into the non-solvent, leading to its entrapment within the crosslinked NG. This methodology allows the production of NGs containing an anticancer drug in one single step, thus avoiding tedious post-encapsulation and purification processes. In addition, the incorporation of the drug during the nanoprecipitation process enabled higher encapsulation efficiencies. Thus, the maximum amount of DOX that was reached when the drug was post-encapsulated within unloaded NGs was $5 \%$ (weight $\%$, data not shown), while values of $10 \%$ could be achieved by the one-pot approach.

DOX-loaded NGs were characterized by FT-IR and DLS. DLS showed a slight increase in size (115 $\mathrm{nm})$ and a low PDI (0.12). In the case of the control NGs that lacked one of the responsive moieties, sizes and PDIs varied significantly (Table 1). DOXloaded NGs were purified using a centrifugal filter device followed by dialysis in PB buffer $10 \mathrm{mM} \mathrm{pH}$ 8. After purification, NGs were analysed by size exclusion chromatography using Sephadex G25 gel to verify that there was no free drug. The NGs were freeze-dried and the DOX content was determined by UV-Vis measurements in DMSO $/ \mathrm{H}_{2} \mathrm{O}(9: 1)(\lambda=503 \mathrm{~nm})$ after performing a calibration curve (Fig. S4, ESI $\dagger$ ). In the case of NGs containing all monomers, DOX quantification can also be performed in $\mathrm{H}_{2} \mathrm{O}$ (Fig. S6a, ESI $\dagger$ ). Notably, DOX loading in the different NGs varied significantly. For example, the NG lacking BMADS displayed a much lower drug content. This might be explained by the less compact polymeric network for this NG, which would have facilitated the diffusion of the drug during the purification steps.

In order to prove DOX stability under free radical polymerization environment, free DOX was treated under NG formation conditions, purified, and analysed by HPLC and mass spectrometry. The expected mass $\left([\mathrm{M}+\mathrm{Na}+2 \mathrm{H}]^{+}: 568.14\right)$ was found in the mass spectrum, while HPLC chromatograms revealed the same retention time for treated-DOX, untreated DOX.HCl, and deprotonated untreated-DOX (Fig. S5, ESI $\dagger$ ).

In vitro DOX release was analysed for the NGs at different $\mathrm{pH}$ values and in the presence of the reducing agent DTT. Notably, an accelerated drug release for the NG containing all monomers and crosslinkers was observed at $\mathrm{pH} 5$ in the presence of $20 \mathrm{mM}$ DTT. Thus, approx. 95\% of DOX was released after $8 \mathrm{~h}$, while only $20 \%$ of free drug could be observed for the same incubation time at $\mathrm{pH} 8$ (Fig. S6b, ESI $\dagger$ ).

The cytotoxicity and internalization of the NGs in A549 lungcarcinoma cells was then investigated. MTT assays showed an $\mathbf{I C}_{50}$ value of $c a .1 \mathrm{mg} \mathrm{mL}^{-1}$ for unloaded NGs (Fig. S7a, ESI $\dagger$ ). However, no effect on cell proliferation and morphology was observed in real time cell analysis (RTCA, Fig. S8, ESI $\dagger$ ) at a concentration of $0.54 \mathrm{mg} \mathrm{mL}^{-1}$, which corresponds to $100 \mu \mathrm{M}$ DOX in the drugloaded NGs. Considering the high cytotoxicity of DOX at such concentration, the NGs appear to be suitable drug carrier candidates.

Cell proliferation inhibition of DOX-loaded NGs was studied by RTCA and MTT assays and finally compared to free DOX

Table 1 Features of DOX-loaded NGs

\begin{tabular}{llll}
\hline Nanogel & Size $(\mathrm{nm})$ & PDI & DOX loading (wt\%) \\
\hline All monomers & 115 & 0.12 & 10 \\
w/o DMDEA & 287 & 0.4 & 10 \\
w/o OEGA & 146 & 0.29 & 8 \\
w/o BMADS & 186 & 0.35 & 7
\end{tabular}




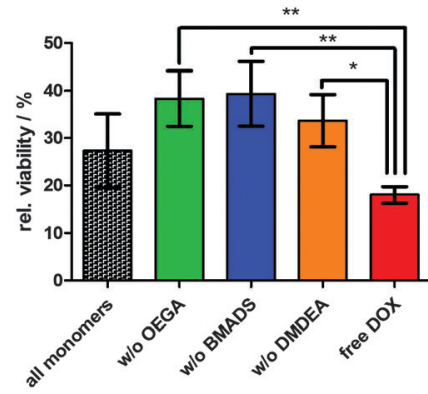

Fig. 3 Cell proliferation inhibition of DOX-loaded NGs at $10 \mu \mathrm{M}$ (DOX concentration) as determined by MTT assay on A549 cells, ${ }^{*} p<0.05$, ${ }^{* *} p<0.01$ ). See also (Table S2, ESI $\dagger$ ).

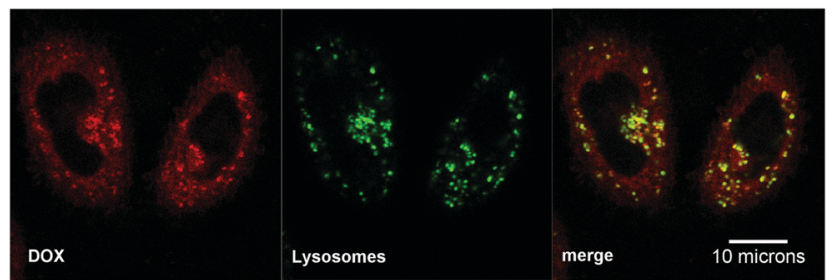

Fig. 4 Confocal microscopy images of DOX-loaded NGs on A549 cells. Red DOX, green: LysoTracker green stained lysosomes, yellow: merged colour.

(Fig. 3, Fig. S7b and S8, ESI $\dagger$ ). Fig. 3 shows the inhibition of cell proliferation at a concentration of $10 \mu \mathrm{M}$ DOX (for doseresponse curves see Fig. S7b, ESI $\dagger$ ). DOX-loaded NGs containing all monomers caused a reduction of viability to $27 \%$ on average, which was the closest to free DOX (18\%). NGs without OEGA, BMADS or DMDEA caused significantly less reduction, with mean values of $38 \%, 39 \%$ and $33.6 \%$, respectively. When the effect of the DOX-loaded NGs on the morphology and proliferation of cancer cells was analysed in real time, the NGs with all monomers showed again the most similar profile to that of free DOX (Fig. S8, $\mathrm{ESI} \dagger$ ). The NGs lacking any of the responsive moieties were less effective and presented very dissimilar profiles, suggesting that the combination of the different stimuli in the multiresponsive NGs may have a synergistic effect.

Finally, internalization of the DOX-loaded NGs (all monomers) into A549 cells was studied by confocal laser scanning microscopy (Fig. 4 and Fig. S9, ESI $\dagger$ ). After 2 h of incubation, the NGs were accumulated in the endosomes/lysosomes (co-localization with LysoTracker), and some DOX fluorescence could be detected in the cytoplasm.

We have developed a simple, reproducible, one-pot synthetic approach that allows the preparation of DOX-loaded multiresponsive NGs based on biocompatible hPG. This strategy combines free radical polymerization, nanoprecipitation, and high-loading drug encapsulation in a single step. The method here described could be applied for the in situ encapsulation of other drugs or bioactive molecules into NGs prepared by polymerization processes. Unloaded NGs were first prepared and their multiresponsive properties were analysed in vitro and compared with the corresponding controls. The NGs showed a high increase in their sizes and PDI in the presence of acidic $\mathrm{pH}$ and DTT, which can be exploited for intracellular drug release. DOX-loaded NGs (containing all monomers or lacking any of the responsive moieties) were prepared and their relative performances in cell proliferation inhibition were studied. The different cytotoxicity profiles obtained in MTT assays and RTCA suggest the presence of a synergistic effect for the multiresponsive NGs. Finally, confocal microscopy studies revealed that DOX-loaded NGs were efficiently internalized by A549 cancer cells.

This research was financially supported by a Marie Curie IEF (Project 302717), the ThermoNanogele NanoMatFutur award (13N12561), and the focus area Nanoscale of the FU Berlin.

\section{Notes and references}

1 O. C. Farokhzad and R. Langer, Adv. Drug Delivery Rev., 2006, 58, 1456-1459.

2 D. Peer, J. M. Karp, S. Hong, O. C. Farokhzad, R. Margalit and R. Langer, Nat. Nanotechnol., 2007, 2, 751-760.

3 Y. Qiu and K. Park, Adv. Drug Delivery Rev., 2001, 53, 321-339.

4 M. Motornov, Y. Roiter, I. Tokarev and S. Minko, Prog. Polym. Sci., 2010, 35, 174-211.

5 S. Ganta, H. Devalapally, A. Shahiwala and M. Amiji, J. Controlled Release, 2008, 126, 187-204.

6 N. A. Peppas, J. Z. Hilt, A. Khademhosseini and R. Langer, Adv. Mater., 2006, 18, 1345-1360.

7 J. K. Oh, R. Drumright, D. J. Siegwart and K. Matyjaszewski, Prog. Polym. Sci., 2008, 33, 448-477.

8 M. Asadian-Birjand, A. Sousa-Herves, D. Steinhilber, J. C. Cuggino and M. Calderon, Curr. Med. Chem., 2012, 19, 5029-5043.

9 A. V. Kabanov and S. V. Vinogradov, Angew. Chem., Int. Ed., 2009, 48, 5418-5429.

10 M. H. Smith and L. A. Lyon, Acc. Chem. Res., 2011, 985-993.

11 X. Zhang, K. Achazi, D. Steinhilber, F. Kratz, J. Dernedde and R. Haag, J. Controlled Release, 2014, 174, 209-216.

12 D. Steinhilber, M. Witting, X. Zhang, M. Staegemann, F. Paulus, W. Friess, S. Küchler and R. Haag, J. Controlled Release, 2013, 169, 289-295.

13 M. Giulbudagian, M. Asadian-Birjand, D. Steinhilber, K. Achazi, M. Molina and M. Calderón, Polym. Chem., 2014, 5, 6909-6913.

14 D. Wilms, S.-E. Stiriba and H. Frey, Acc. Chem. Res., 2010, 43, 129-141.

15 M. Calderón, M. A. Quadir, S. K. Sharma and R. Haag, Adv. Mater., 2010, 22, 190-218.

16 R. K. Kainthan and D. E. Brooks, Biomaterials, 2007, 28, 4779-4787.

17 M. Schömer, C. Schüll and H. Frey, J. Polym. Sci., Part A: Polym. Chem., 2013, 51, 995-1019.

18 L. Zha, B. Banik and F. Alexis, Soft Matter, 2011, 7, 5908-5916.

19 S. Lee, K. Saito, H.-R. Lee, M. J. Lee, Y. Shibasaki, Y. Oishi and B.-S. Kim, Biomacromolecules, 2012, 13, 1190-1196.

20 Z.-Y. Qiao, R. Zhang, F.-S. Du, D.-H. Liang and Z.-C. Li, J. Controlled Release, 2011, 152, 57-66.

21 J. Peng, T. Qi, J. Liao, B. Chu, Q. Yang, W. Li, Y. Qu, F. Luo and Z. Qian, Biomaterials, 2013, 34, 8726-8740.

22 S. Mura, J. Nicolas and P. Couvreur, Nat. Mater., 2013, 12, 991-1003.

23 E. Lepeltier, C. Bourgaux and P. Couvreur, Adv. Drug Delivery Rev., 2014, 71, 86-97.

24 Z.-Y. Qiao, F.-S. Du, R. Zhang, D.-H. Liang and Z.-C. Li, Macromolecules, 2010, 43, 6485-6494.

25 S. Höfl, L. Zitzler, T. Hellweg, S. Herminghaus and F. Mugele, Polymer, 2007, 48, 245-254.

26 G. Liu, Q. Qiu and Z. An, Polym. Chem., 2012, 3, 504-513. 